\title{
TDP-43 as a potential biomarker for amyotrophic lateral sclerosis: a systematic review and meta-analysis
}

\author{
Vivek Majumder ${ }^{1 \dagger}$, Jenna M. Gregory ${ }^{1,2^{*}}$ (D), Marcelo A. Barria ${ }^{3}$, Alison Green ${ }^{3}$ and Suvankar Pal ${ }^{1,2,3^{*}}$
}

\begin{abstract}
Background: Frontotemporal dementia (FTD) and Amyotrophic Lateral Sclerosis (ALS) are incurable, progressive and fatal neurodegenerative diseases with patients variably affected clinically by motor, behavior, and cognitive deficits. The accumulation of an RNA-binding protein, TDP-43, is the most significant pathological finding in approximately $95 \%$ of ALS cases and 50\% of FTD cases, and discovery of this common pathological signature, together with an increasing understanding of the shared genetic basis of these disorders, has led to FTD and ALS being considered as part of a single disease continuum. Given the widespread aggregation and accumulation of TDP-43 in FTD-ALS spectrum disorder, TDP-43 may have potential as a biomarker in these diseases.

Methods: We therefore conducted a systematic review and meta-analysis to evaluate the diagnostic utility of TDP-43 detected in the cerebrospinal fluid (CSF) of patients with FTD-ALS spectrum disorder.

Results: From seven studies, our results demonstrate that patients with ALS have a statistically significantly higher level of TDP-43 in CSF (effect size 0.64, 95\% Cl: $0.1-1.19, p=0.02$ ).
\end{abstract}

Conclusions: These data suggest promise for the use of CSF TDP-43 as a biomarker for ALS.

Keywords: TDP-43, Amyotrophic lateral sclerosis, Biomarker, Systematic review, Meta-analysis

\section{Background}

Frontotemporal dementia (FTD) is an umbrella term for a spectrum of neurodegenerative disorders. These disorders primarily affect behaviour, personality, executive function and language (and may include motor impairment). It is the second most common form of dementia after Alzheimer's disease $(\mathrm{AD})$ in people under 65 years old, with the average age of onset being between 45 and 65 years old, and the peak prevalence being around age 65 to 69 [1]. Prognosis is poor with a median survival of $8-10$ years and a steady rate of decline [2]. Currently, there are no curative or disease modifying treatments (DMT) available, so patients are treated symptomatically [3].

FTD also affects the motor system and is now increasingly thought to be on a syndromic spectrum with amyotrophic lateral sclerosis (ALS; [4]). ALS is a

\footnotetext{
* Correspondence: jenna.gregory@IGMM.ed.ac.uk; suvankar.pal@ed.ac.uk † ivek Majumder and Jenna M. Gregory contributed equally to this work. ${ }^{1}$ Centre for Clinical Brain Sciences, University of Edinburgh, Chancellor's Building, Edinburgh EH16 4SB, UK

Full list of author information is available at the end of the article
}

neurodegenerative disease, and has a heterogeneous presentation affecting motor function, eventually affecting the ability to speak, swallow and breathe [5]. It affects both upper and lower motor neurons, but tends to spare sensory neurons. Sporadic cases of ALS have a peak incidence at around 60 years old, whereas familial cases peak at 4352 years old. Familial cases make up 10-20\% of cases [5]. Crucially, approximately $50 \%$ of patients exhibit changes in behaviour and cognition $[1,5-7]$, with up to $15 \%$ developing FTD [8-10]. Median survival from disease onset is 3.5 years and from diagnosis is 2 years [8], though $5-10 \%$ of patients can survive up to 10 years or more [6]. Riluzole, a sodium channel blocker which may also have other effects through inhibition of NMDA receptor signaling, glutamate release and uptake, is the only licensed DMT for ALS, and has been demonstrated to prolong life by 34 months [11]. There have been recent reports suggesting efficacy of Edaravone and Masitinib as additional treatments but these drugs have yet to receive widespread licensing. Mean time to diagnosis is 15 months [8] from the 
onset of first symptoms (ranging from 5 to 24) and relies on clinical evaluation, supported by examination and electromyography. This long diagnostic delay reflects the diagnostic challenge faced when assessing patients with a clinically heterogeneous and progressive neurodegenerative disease. The lack of robust biomarkers and diagnostic tests to support an accurate diagnosis further compounds this diagnostic challenge. The identification of biomarkers will enable early and accurate diagnosis thus avoiding unnecessary investigations and enable rapid stratification of patients into clinical trials. There are currently no biomarkers for FTD or ALS.

A substantial monogenetic component to these diseases has recently been established. The most frequent genetic abnormality is an intronic hexanucleotide repeat expansion in the gene that codes for C9orf72, found to be present in the majority of cases of familial cases of FTD-ALS spectrum disorders $[4,6]$. Pathological assessment of specific brain regions of these cases at post-mortem reveals characteristic immuno-histochemical staining patterns of abnormally phosphorylated TDP-43 in cytoplasmic inclusion (particularly in the motor cortex, spinal cord and the frontal and temporal lobes) and p62 accumulation (particularly in the granule cell layer of the cerebellum and in the dentate gyrus of the hippocampus). In 2006, TDP-43 was identified as the main pathological finding in most sporadic and familial cases of both ALS and FTD [12]. TDP-43 is a protein with multiple functions, but is primarily involved in alternative splicing and transcriptional regulation [6]. In FTD and ALS, TDP-43 becomes ubiquinated, hyperphosphorylated and C-terminally truncated, increasing its aggregation propensity and causing widespread neurotoxicity and cell death [1]. Given the high burden of TDP-43 accumulation in the central nervous system of the majority of patients with FTD-ALS spectrum disorder, TDP-43 has been postulated as a biomarker in this disease.

A pathological diagnosis (rather than a clinical diagnosis) of FTD is referred to as frontotemporal lobar degeneration (FTLD). Indeed, three major pathological subtypes of FTLD exist characterized by the type of pathology observed in post-mortem tissue. FTLD- tau, FTLD-FUS and FTLD-TDP. These molecular subtypes are characterized respectively by the accumulation of misfolded tau, FUS and TDP-43 intracellular inclusions [13]. Due to the increasing prevalence of DMTs targeting specific molecular mechanisms there is an increasingly emergent need for biomarkers to distinguish between these subtypes. A potential biomarker, such as CSF TDP-43 could have the potential to predict the neuropathological diagnosis and thus provide us, not only with a diagnostic biomarker, but also a biomarker to enable stratification for targeted DMT development.

The aims of this study are to systematically review all studies analysing CSF TDP-43 concentrations in FTD and ALS patients and conduct a meta-analysis to investigate whether there is a significant difference between concentrations of CSF TDP-43 in patients with FTD-ALS spectrum disorders compared to neurological and non-neurological controls. Our hypothesis is that CSF TDP-43 will be significantly increased in patients with FTD-ALS spectrum disorders compared to controls.

\section{Methods \\ Objectives \\ Population}

Clinical studies of patients with FTD-ALS spectrum disorder (ALS, FTD, and FTD-ALS).

\section{Intervention \\ CSF TDP-43 detected by ELISA or western blot.}

\section{Comparison}

Control patients who are 1) Neurological controls (diagnosed with non-dementia neurological conditions), or 2) Non-neurological controls (diagnosed with a non-neurological condition or healthy).

\section{Outcome measure}

Primary outcome: effect size (concentration of TDP-43 in CSF of in FTD-ALS, FTD and ALS patients compared to controls). Secondary outcomes: Assessment of quality and heterogeneity between studies.

\section{Study design}

All study types where CSF TDP-43 concentrations were measured and compared to a control.

\section{Search methods \\ Sources}

Databases: 1. PubMed, 2. Medline, 3. EMBASE 4. LILACs 5. IMEMR 6. WPRIM 7. Chinese Science Citation Index.

The date of searches was $01 / 03 / 17$ and there were no publication date restrictions and no language restrictions.

\section{Search terms}

(Frontotemporal Lobe Dementia [MeSH] OR Amyotrophic Lateral Sclerosis [MeSH] OR Motor Neuron Disease $[\mathrm{MeSH}]$ ) AND (Cerebrospinal Fluid [MeSH] OR Lumber Puncture [MeSH]) AND TDP [MeSH]

\section{Screening}

The title and abstract of each paper were screened for relevance with respect to inclusion and exclusion criteria. For studies meeting our predefined criteria the full text was retrieved, imported to Excel and duplicate records were discarded. The quality of each paper was then evaluated by the QUADAS-2 quality score and relevant data were extracted. 


\section{Eligibility}

Inclusion criteria

- All studies that measure TDP-43 in the CSF of living patients with ALS or FTD, compared to controls.

\section{Exclusion criteria}

- No control group

- Animal studies

- Post mortem studies

- Reviews

- Letters and comments

- Abstracts

- Non-parametric results (Meta-Analysis only)

Study characteristics to be extracted:

- Study ID: (i) Author and (ii) Year

- Intervention: FTD (behavioural, non-fluent or semantic), ALS or both (FTD-ALS)

- Medical diagnosis of comparison/controls

- Intervention quantification method (western blot or ELISA)

- Age: mean age of patients and controls

- Sex: of patients and controls

- Diagnosis method of patients

- Genetic factors

- Sample size: of patients and controls

\section{Further searches}

Two similar searches were done, with the similar objectives. These were to look at the effect size of lymphocytic bound TDP-43 in FTD, ALS and FTD-ALS patients compared to controls, and the effect size of plasma TDP-43 in FTD, ALS and FTD-ALS compared to controls. However, neither of these searches produced enough papers in order to do a systematic review.

\section{Search terms}

(Frontotemporal Lobe Dementia [MeSH] OR Amyotrophic Lateral Sclerosis $[\mathrm{MeSH}]$ OR Motor Neuron Disease [MeSH]) AND (Lymphocyte [MeSH] OR White Cell [MeSH] or White Blood Cell [MeSH]) AND TDP [MeSH].

(Frontotemporal Lobe Dementia [MeSH] OR Amyotrophic Lateral Sclerosis [MeSH] OR Motor Neuron Disease $[\mathrm{MeSH}]$ ) AND (Plasma [MeSH] OR Serum [MeSH] OR Blood [MeSH]) AND TDP [MeSH]

\section{Statistical analysis}

An individual meta-analysis was carried out for each group of patients (i) FTD alone, (ii) ALS alone and (iii) FTD and ALS patients combined as an overall evaluation of FTD-ALS spectrum disorders. Additional subgroup analyses included: an assessment of heterogeneity and a quality score. Outcome measures were calculated for each of the studies identified and included on a forest plot. Given the variability of intervention quantification included in the analysis, outcome measures were recorded in standardised mean differences (SMD), to allow for meaningful comparisons between studies. SMD was compared using Hedges g-statistic, to account for bias from small sample sizes, using a random effects model. SMDs were reported as odds ratios with $95 \%$ confidence intervals. Heterogeneity was assessed for all outcome measures using $\mathrm{I}^{2}$-values, and a funnel plot and Egger's regression test was used to assess publication bias.

\section{Results}

Systematic review reveals poor reporting of measures to reduce bias but no evidence of publication bias

Overall, 6 studies were identified for meta-analysis, including a total of 274 participants [14-19], 150 neurological controls (Table 1) and 146 patients with FTD-ALS spectrum disorders (94 with ALS and 77 with FTD) (Fig. 1a). Seven studies were included in the qualitative analysis (Table 1; Fig. 1a). The data from the Suarez-Calvet et al., 2014 study [20] was non-parametric, so could not be compared to the other studies in the quantitative analysis. There was no evidence of publication bias detected by Egger's regression (best-fit gradient of $-0.01017 \pm 0.03182$ and $p=0.8029$ ) or funnel plot. Systematic review, risk of bias and quality assessment revealed that only 3 of the 7 identified papers reported on measures to reduce bias, such as blinding (Table 1). Flve of the studies used an ELISA technique to evaluate the concentration of TDP-43 in CSF samples [14-18] with the remaining two papers using a semi-quantitative analysis of immunoblot intensity. Suarez-Calvet et al., 2014 report concentrations as an ELISA comparison, with a manufactured TDP-43 standard. Steinacker et al., 2008 used an immunoblot intensity comparison between samples and a stock sample of CSF TDP-43 from the post mortem frontal lobe of a single FTD patient, which increases the risk of bias due to the semi-quantitative nature of immunoblotting. Four of the 7 studies were assessed as having a low risk of bias as determined by QUADAS-2 quality assessment (Table 1; [14-16, 19]) and 2 studies were assessed to have a risk of bias due to lack of blinding $[17,18]$. The control populations used were different between studies, 2 studies used healthy controls $[19,20]$ and 4 used non-neurodegenerative neurological patients as controls $[14,16-18]$ with conditions such as focal complex seizures or depression. Kasai et al., used a combination of non-neurodegenerative neurological patients and healthy controls. All studies used an appropriate clinical diagnosis to confirm the diagnosis of ALS or FTD. Two studies used additional, supportive diagnostic modalities such as fMRI or post mortem lab testing $[14,18]$. 


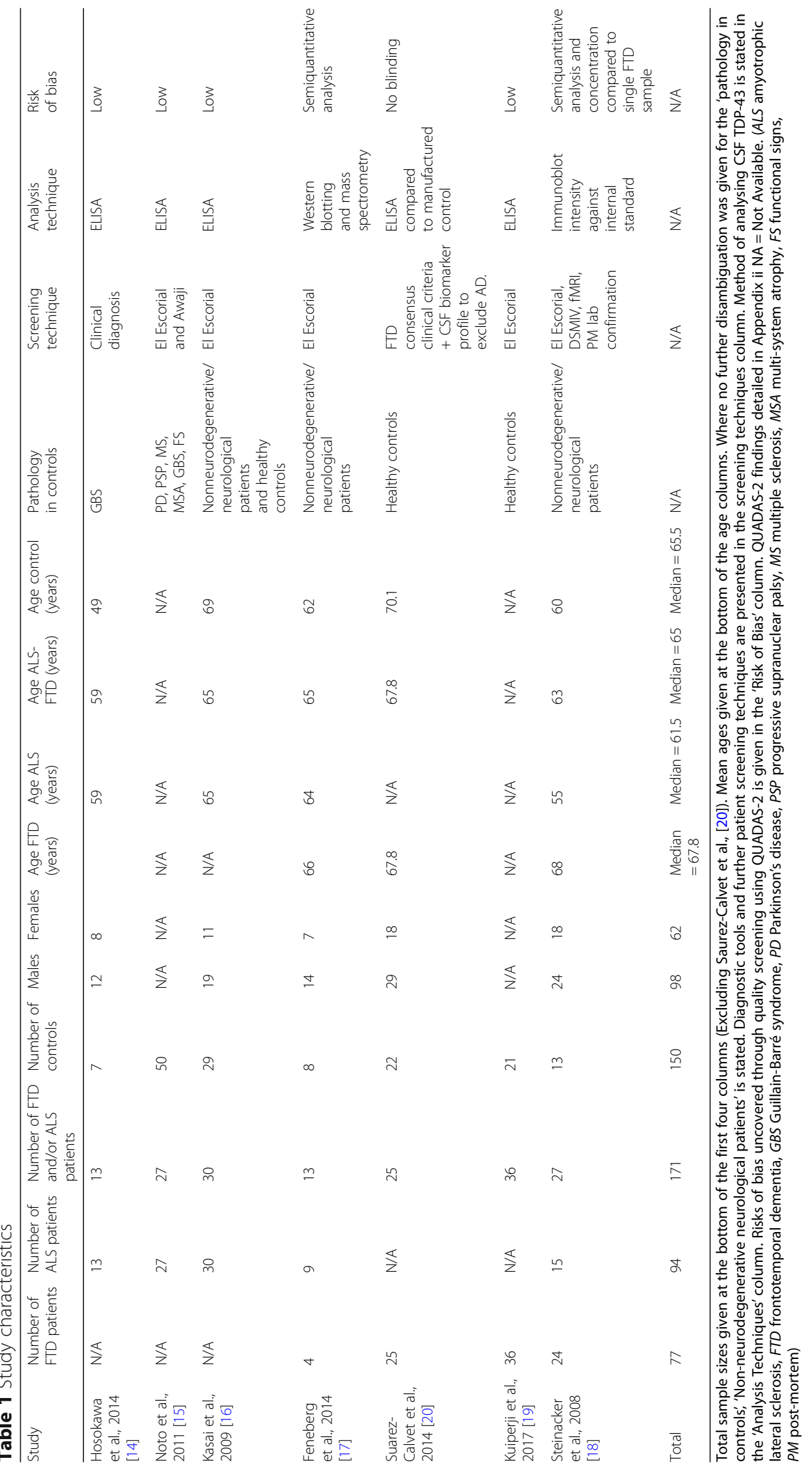




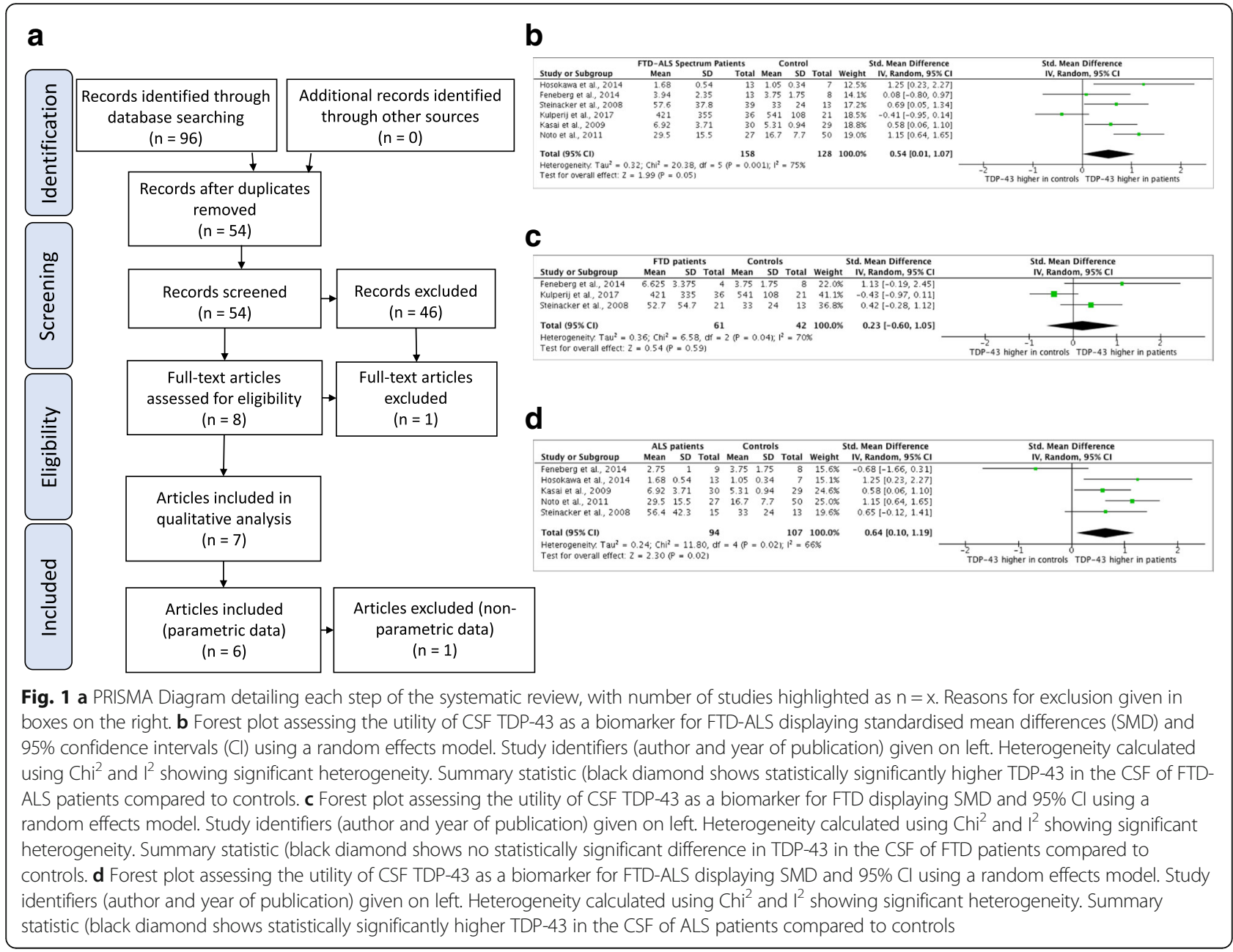

Meta-analysis reveals a statistically significant increase in TDP-43 in the CSF of FTD-ALS and ALS patients, but not FTD patients alone

We conducted three separate meta-analyses of the included studies to evaluate the potential of CSF TDP-43 as a biomarker in each of (i) FTD-ALS spectrum disorders (ALS and FTD combined), (ii) FTD alone, and (iii) ALS alone. Meta-analysis of FTD-ALS spectrum disorders versus controls using standard mean difference found an effect estimate of 0.55 (95\% CI: 0.01-1.09) and a Z-score of $2.0(p<0.05)$ demonstrating a significant increase in detectable TDP-43 in the CSF of patients with FTD or ALS compared to controls. However, $\mathrm{Chi}^{2}$ was 20.57 and $\mathrm{I}^{2}$ was $76 \%$ showing significant heterogeneity in this dataset (Fig. 1b). Meta-analysis of studies assessing TDP-43 in the CSF of patients with FTD alone showed an effect estimate of 0.50 (95\% CI: $-0.65-1.65$; Fig. 1c), although this favours increased levels of TDP-43 in the control group, this finding does not reach statistical significance with a Z-score of $0.85(p=0.40)$. There was a similarly significant heterogeneity with a $\mathrm{Chi}^{2}$ of 10.75 and an $\mathrm{I}^{2}$ of $81 \%$. Meta-analysis of studies assessing TDP-43 in the CSF of patients with ALS alone showed an effect estimate of 0.64 (95\% CI: 0.10-1.19). A Z-score of $2.3(p=0.02)$ showing a significant increase in detectable TDP-43 in the CSF of ALS patients. Chi ${ }^{2}$ was 11.80 and $\mathrm{I}^{2}$ was $66 \%$ showing moderate heterogeneity (Fig. 1d).

\section{Discussion}

To our knowledge, this is the first systematic review and meta-analysis of the literature assessing the utility of TDP-43 in the CSF of patients with FTD-ALS spectrum disorders as a potential diagnostic biomarker. We have shown that CSF TDP-43 is significantly increased in patients with FTD-ALS spectrum disorder. However, when analysing patients with ALS and FTD separately, only patients with ALS (not patients with FTD) showed a significantly increased CSF TDP-43. We also detected substantial heterogeneity and relative risk of bias in these studies. This heterogeneity is likely due to the wide variety of methods used to analyse CSF TDP-43 concentrations, but could also reflect the disease heterogeneity (both clinically and genetically) seen in these spectrum 
disorders and the heterogeneity of controls used for comparison. Two of the studies used normal healthy controls $[19,20]$ whilst the remaining included patients with a wide variety of neurological diagnoses as controls. Kuiperij et al. excluded patients with non-FTD-ALS motor deficits, meaning their data could attenuate the effect size of FTD-ALS spectrum and pure FTD patients in this meta-analysis, therefore increasing heterogeneity. Steinacker et al. compared their concentration of TDP-43 to a standard developed from a single patient with FTD. There is no indication of how representative this patient is compared to other FTD-ALS spectrum disorder patients and depending on the concentration of TDP-43 in their standard, this could affect the final reported TDP-43 concentrations. Risk of bias could also have had an impact on the heterogeneity seen. For example, neither Feneberg et al. nor Suarez-Calvet et al. commented on blinding in their methods, an important aspect of scientific rigor to ensure that all samples are treated and assessed equally, limiting the potential for bias. Furthermore, variation in time from sampling to storage, from 30 min to several hours (and in 5 out of 7 studies, this time was not documented), post CSF sampling may influence TDP-43 quantification due to protein degradation. Diagnostic criteria for patient identification did not vary between studies, all but two studies used the El Escorial criteria for ALS and all studies studying FTD used Consensus Criteria for FTD, and many of the studies went to further investigate a diagnosis of FTD, ALS or FTD-ALS by with more detailed imaging studies or post mortem [18].

Given the significant effect size shown in this meta-analysis, demonstrating an increase in the levels of detectable TDP-43 in patients with ALS, further research to refine the use of CSF TDP-43 as a diagnostic tool is warranted. Furthermore, a longitudinal study of CSF TDP-43 concentrations in patients with FTD-ALS spectrum disorders might show if CSF TDP-43 increases with disease progression. Similarly, a longitudinal study investigating carriers of the C9orf72 hexanucleotide expansion might provide an indication as to how early TDP-43 aggregation occurs, and potentially would allow us to diagnose FTD-ALS spectrum disorders pre-symptomatically in these patients $[4,7,21]$. This would facilitate study of preventative and disease modifying interventions in early disease. However, given the wide range of techniques used to evaluate the concentration of TDP-43 in FTD-ALS spectrum disorder patients there clearly needs to be agreement with regards to consistency of sampling, storage and testing techniques, with a standardized operating protocol adhered to by all diagnostic laboratories. Ideally, a larger, much more highly powered study into the effect of CSF TDP-43 as a diagnostic tool for FTD-ALS spectrum disorders would be done. This study should have aged-matched controls and ALS, FTD and crucially FTD-ALS patients (patients with both ALS and FTD symptoms), with the controls having no neurological or psychiatric morbidity and the patients having no confounding co-morbidities or genetic variations (such as C9orf72) that might skew data. The study should be carried out longitudinally in order to map the progression of TDP-43 in both controls and patients. Diagnosis would be done using currently approved diagnostic tools, with post-mortem pathological verification of diagnosis. Both the sampler and the pathologist should be blinded to whether the CSF is from a control or a patient. Equally, analysis should be done with an ELISA and presented in empirical concentrations to improve transparency. As of yet, there are not enough studies into the TDP-43 in the blood, either in lymphocytes or in plasma. However, these are avenues for future meta-analyses (given more studies), as they provide less invasive diagnostic tests than lumbar puncture.

\section{Study limitations}

Our data demonstrate a significant difference in TDP-43 levels in ALS patients compared to controls, implying that CSF TDP-43 levels show promise as a potential biomarker in ALS. It is probable that the statistically significant result observed in FTD-ALS spectrum disorder cases (Fig. 1b), could be due to the ALS patients included therein as FTD patients alone did not show a statistically significant increase in CSF TDP-43. This is likely reflected in the substantial heterogeneity seen in the combined analysis. However, whilst the lower confidence interval for FTD alone cases does intersect the line of no effect, the effect size and upper confidence interval are still favouring a higher level of TDP-43 in cases compared to controls, indicating that further studies are warranted to reduce the confidence intervals sufficiently to ascertain the potential of TDP-43 as a biomarker in FTD. Furthermore, whilst TDP-43 pathology is a unifying feature of the majority of ALS and FTD cases, TDP-43 accumulation is also observed in other neurodegenerative conditions; including but not limited to $\mathrm{AD}$. Whilst our findings suggest that CSF TDP-43 may be a promising biomarker in ALS, it's potential in the context of other neurodegenerative conditions is not yet fully understood. There are currently only two publications assessing the utility of TDP- 43 as a biomarker in $\mathrm{AD}[22,23]$, which we considered to be too small a sample to conduct a meta-analysis on. However, future systematic reviews should consider including an assessment of TDP-43 in other neurodegenerative diseases when these data become available.

\section{Conclusion}

Our systematic review and meta-analysis reveals early supportive data indicating that TDP-43 detected in the CSF of patients with FTD-ALS spectrum disorders in particular ALS patients could be a promising biomarker in these diseases. Given the current paucity of diagnostic and prognostic biomarkers in these disorders, this result clearly indicates that further studies in to its utility are warranted. 


\section{Abbreviations}

AD: Alzheimer's disease; ALS: Amyotrophic lateral sclerosis; CSF: Cerebrospinal fluid; DMT: Disease modifying treatments; ELISA: Enzyme-linked immunosorbent assay; FID: Fronto-temporal dementia; TDP-43: TAR-DNA binding protein 43 kDa

\section{Funding}

The work was funded by the Future-MND grant. Grant code: 217ARF R43969, 3138. Grant holder SP

\section{Availability of data and materials}

All data are available within the text of the manuscript.

\section{Authors' contributions}

$V M, J G, M A B, A G$ and $S P$ contributed to writing and reviewing data and screening articles. VM, JMG, MAB and AG and SP contributed to data extraction and analysis of the results. JG and SP supervised and conceptualized the project. Specifically, all authors have (i) made substantial contributions to conception and design, or acquisition of data, or analysis and interpretation of data; (ii) been involved in drafting the manuscript or revising it critically for important intellectual content; (iii) given final approval of the version to be published; and (iv) agreed to be accountable for all aspects of the work in ensuring that questions related to the accuracy or integrity of any part of the work are appropriately investigated and resolved.

\section{Ethics approval and consent to participate} N/A

\section{Consent for publication}

N/A

\section{Competing interests}

The authors declare that they have no competing interests.

\section{Publisher's Note}

Springer Nature remains neutral with regard to jurisdictional claims in published maps and institutional affiliations.

\section{Author details}

${ }^{1}$ Centre for Clinical Brain Sciences, University of Edinburgh, Chancellor's Building, Edinburgh EH16 4SB, UK. ²Euan MacDonald Centre for Motor Neurone Disease Research, University of Edinburgh, Edinburgh, UK. ${ }^{3}$ National CJD Research and Surveillance Unit, Bryan Matthews Building, Western General Hospital, Crewe Rd, Edinburgh EH4 2XU, UK.

\section{Received: 27 February 2018 Accepted: 14 June 2018}

Published online: 28 June 2018

\section{References}

1. Olney NT, Spina S, Miller BL. Frontotemporal Dementia. Neurol Clin. 2017; 35(2):339-74.

2. Harding M. Frontotemporal Dementia: Patient.info/pro; 2014 [updated 19/9/ 2014. Available from: https://patient.info/doctor/frontotemporal-dementia.

3. Tsai RM, Boxer AL. Therapy and clinical trials in frontotemporal dementia: past, present, and future. J Neurochem. 2016;138(Suppl 1):211-21.

4. Couratier P, Corcia P, Lautrette G, Nicol M, Marin B. ALS and frontotemporal dementia belong to a common disease spectrum. Rev Neurol (Paris). 2017; 173(5):273-9.

5. Soriani $\mathrm{MH}$, Desnuelle $\mathrm{C}$. Care management in amyotrophic lateral sclerosis. Rev Neurol (Paris). 2017:173(5):288-99.

6. Rademakers R, Neumann M, Mackenzie IR. Advances in understanding the molecular basis of frontotemporal dementia. Nat Rev Neurol. 2012;8(8):423-34.

7. Olszewska DA, Lonergan R, Fallon EM, Lynch T. Genetics of frontotemporal dementia. Curr Neurol Neurosci Rep. 2016;16(12):107.

8. Black HA, Leighton DJ, Cleary EM, Rose E, Stephenson L, Colville S, Ross D, Warner J, Porteous M, Gorrie GH, Swingler R, Goldstein D, Harms MB, Connick P, Pal S, Aitman TJ, Chandran S. Genetic epidemiology of motor neuron disease-associated variants in the Scottish population. Neurobiol Aging. 2017;51:178.e11-20.

9. Byrne S, Heverin M, Elamin M, Bede P, Lynch C, Kenna K, MacLaughlin R, Walsh C, Al Chalabi A, Hardiman O. Aggregation of neurologic and neuropsychiatric disease in amyotrophic lateral sclerosis kindreds: a population-based case-control cohort study of familial and sporadic amyotrophic lateral sclerosis. Ann Neurol. 2013:74:699-708.

10. Gibson SB, Figueroa KP, Bromberg MB, Pulst SM, Cannon-Albright L. Familial clustering of ALS in a population-based resource. Neurology. 2014:82(1):17-22.

11. Wagner ML, Landis BE. Riluzole: a new agent for amyotrophic lateral sclerosis. Ann Pharmacother. 1997;31(6):738-44.

12. Neumann M, Sampathu DM, Kwong LK, Truax AC, Micsenyi MC, Chou TT, et al. Ubiquitinated TDP-43 in frontotemporal lobar degeneration and amyotrophic lateral sclerosis. Science. 2006;314(5796):130-3.

13. Perry DC, Brown JA, Possin KL, Datta S, Trujillo A, et al. Clinicopathological correlations in behavioural variant frontotemporal dementia. Brain. 2017; 140(12):3329-45

14. Hosokawa M, Arai T, Yamashita M, Tsuji H, Nonaka T, Masuda-Suzukake M, et al. Differential diagnosis of amyotrophic lateral sclerosis from Guillain-Barre syndrome by quantitative determination of TDP-43 in cerebrospinal fluid. Int J Neurosci. 2014;124(5):344-9.

15. Noto Y, Shibuya K, Sato Y, Kanai K, Misawa S, Sawai S, et al. Elevated CSF TDP-43 levels in amyotrophic lateral sclerosis: specificity, sensitivity, and a possible prognostic value. Amyotroph Lateral Scler. 2011;12(2):140-3.

16. Kasai T, Tokuda T, Ishigami N, Sasayama H, Foulds P, Mitchell DJ, et al. Increased TDP-43 protein in cerebrospinal fluid of patients with amyotrophic lateral sclerosis. Acta Neuropathol. 2009:117(1):55-62.

17. Feneberg $E$, Steinacker $P$, Lehnert S, Schneider A, Walther $P$, Thal DR, et al. Limited role of free TDP-43 as a diagnostic tool in neurodegenerative diseases. Amyotroph Lateral Scler Frontotemporal Degener. 2014;15(5-6):351-6.

18. Steinacker $P$, Hendrich $C$, Sperfeld AD, Jesse S, von Arnim CA, Lehnert S, et al. TDP-43 in cerebrospinal fluid of patients with frontotemporal lobar degeneration and amyotrophic lateral sclerosis. Arch Neurol. 2008;65(11):1481-7.

19. Kuiperij HB, Versleijen AA, Beenes M, Verwey NA, Benussi L, Paterlini A, et al. Tau rather than TDP-43 proteins are potential cerebrospinal fluid biomarkers for frontotemporal lobar degeneration subtypes: a pilot study. J Alzheimers Dis. 2017;55(2):585-95.

20. Suarez-Calvet M, Dols-Icardo O, Llado A, Sanchez-Valle R, Hernandez I, Amer $\mathrm{G}$, et al. Plasma phosphorylated TDP-43 levels are elevated in patients with frontotemporal dementia carrying a C9orf72 repeat expansion or a GRN mutation. J Neurol Neurosurg Psychiatry. 2014;85(6):684-91.

21. Williams SM, Khan G, Harris BT, Ravits J, Sierks MR. TDP-43 protein variants as biomarkers in amyotrophic lateral sclerosis. BMC Neurosci. 2017;18(1):20.

22. Foulds $P$, McAuley E, Gibbons L, Davidson Y, Pickering-Brown SM, Neary D, Snowden JS, Allsop D, Mann DM. TDP-43 protein in plasma may index TDP43 brain pathology in Alzheimer's disease and frontotemporal lobar degeneration. Acta Neuropathol. 2008;116(2):141-6.

23. Williams SM, Schulz P, Rosenberry TL, Caselli RJ, Sierks MR. Blood-based oligomeric and other protein variant biomarkers to facilitate pre-symptomatic diagnosis and staging of Alzheimer's disease. J Alzheimers Dis. 2017;58(1):23-35

\section{Ready to submit your research? Choose BMC and benefit from:}

- fast, convenient online submission

- thorough peer review by experienced researchers in your field

- rapid publication on acceptance

- support for research data, including large and complex data types

- gold Open Access which fosters wider collaboration and increased citations

- maximum visibility for your research: over $100 \mathrm{M}$ website views per year

At BMC, research is always in progress.

Learn more biomedcentral.com/submissions 